\title{
REMETRIZATION RESULTS \\ FOR POSSIBLY INFINITE SELF-SIMILAR SYSTEMS
}

\author{
Radu Miculescu - Alexandru Mihail
}

\begin{abstract}
In this paper we introduce a concept of possibly infinite selfsimilar system which generalizes the attractor of a possibly infinite iterated function system whose constitutive functions are $\varphi$-contractions. We prove that for a uniformly possibly infinite self-similar system there exists a remetrization which makes contractive all its constitutive functions. Then, based on this result, we show that for such a system there exist a comparison function $\varphi$ and a remetrization of the system which makes $\varphi$-contractive all its constitutive functions. Finally we point out that in the case of a finite set of constitutive functions our concept of a possibly infinite self-similar system coincides with Kameyama's concept of a topological self-similar system.
\end{abstract}

\section{Introduction}

In order to generalize the notion of the attractor of an iterated function system A. Kameyama (see [10]) introduced the concepts of topological self-similar set and self-similar topological system as follows:

Definition 1.1. A compact Hausdorff topological space $K$ is called a topological self-similar set if there exist continuous functions $f_{1}, \ldots, f_{N}: K \rightarrow K$, where $N \in \mathbb{N}^{*}=\{1,2, \ldots\}$, and a continuous surjection $\pi: \Lambda \rightarrow K$, where

2010 Mathematics Subject Classification. 28A80, 37B10, 37C70, 54E35.

Key words and phrases. Possibly infinite self-similar systems; remetrizations; $\varphi$-contractions. 
$\Lambda=\{1, \ldots, N\}^{\mathbb{N}^{*}}$, such that the diagram

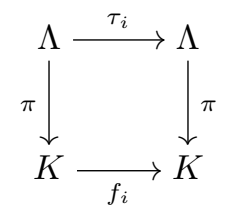

commutes for all $i \in\{1, \ldots, N\}$, where

$\tau_{i}\left(\omega_{1} \ldots \omega_{m} \omega_{m+1} \ldots\right)=i \omega_{1} \ldots \omega_{m} \omega_{m+1} \ldots \quad$ for each $\omega_{1} \ldots \omega_{m} \omega_{m+1} \ldots \in \Lambda$.

We say that $\left(K,\left\{f_{i}\right\}_{i \in\{1, \ldots, N\}}\right)$, a topological self-similar set together with a set of continuous maps as above, is a topological self-similar system.

He asked the following fundamental question (see [10]): Given a topological self-similar system $\left(K,\left\{f_{i}\right\}_{i \in\{1, \ldots, N\}}\right)$, does there exist a metric on $K$ compatible to the topology such that all the functions $f_{i}$ are contractions? Such a metric is called a self-similar metric. L. Janoš ([8] and [9]) settles the case $N=1$.

On the one hand, Kameyama provided a topological self-similar set which does not admit a self-similar metric and, on the other hand, he proved that every totally disconnected self-similar set and every non-recurrent finitely ramified selfsimilar set have a self-similar metric.

R. Atkins, M. Barnsley, A. Vince and D. Wilson [1] gave an affirmative answer to the above question for self-similar sets derived from affine transformations on $\mathbb{R}^{m}$ (see also [12] for a generalization of this result for a Banach space $(X,\|\cdot\|)$ instead of the Banach space $\mathbb{R}^{m}$ and for an arbitrary set $I$ instead of the set $\{1, \ldots, N\})$, M. Barnsley and A. Vince [4] for projectives functions and A. Vince [14] for Möbius transformations.

The problem of the existence of a self-similar metric on a self-similar set was also studied by K. Hveberg [7], M. Barnsley and K. Igudesman [3], T. Banakh, W. Kubiś, N. Novosad, M. Nowak and F. Strobin [2].

In [13], we modified Kameyama's question (which, as we have seen, has a negative answer for an arbitrary topological self-similar system) by weakening the requirement that the functions in the topological self-similar system are contractions to requiring that they are $\varphi$-contractions. More precisely, we gave an affirmative answer to the following question: Given a topological self-similar system $\left(K,\left\{f_{i}\right\}_{i \in\{1, \ldots, N\}}\right)$, does there exist a metric $\delta$ on $K$ which is compatible with the original topology and a comparison function $\varphi$ such that $f_{i}:(K, \delta) \rightarrow$ $(K, \delta)$ is $\varphi$-contraction for each $i \in\{1, \ldots, N\}$ ?

In this paper we study the case of a possibly infinite family of functions $\left(f_{i}\right)_{i \in I}$. We introduce the concept of possibly infinite self-similar system which generalizes the notion of the attractor of a possibly infinite iterated function system whose constitutive functions are $\varphi$-contractions (see Proposition 3.7). 
We prove that for a uniformly possibly infinite self-similar system there exists a remetrization which makes contractive all its constitutive functions (see Theorem 4.1). Then, based on this result, we show that for such a system there exist a comparison function $\varphi$ and a remetrization of the system which makes $\varphi$ contractive all its constitutive functions (see Theorem 5.5). Finally we point out that when the set $I$ is finite the concepts of a possibly infinite self-similar system and a topological self-similar system coincide. Consequently we obtain a generalization of the above mentioned affirmative answer to modified Kameyama's question.

\section{Preliminaries}

In the sequel, by $\mathbb{N}$ we mean the set $\{0,1, \ldots\}$ and by $\mathbb{N}^{*}$ the set $\{1,2, \ldots\}$. Let $I$ be an arbitrary set. By $\Lambda(I)$ we mean the set $I^{\mathbb{N}^{*}}$ and by $\Lambda_{n}(I)$ we mean the set $I^{\{1, \ldots, n\}}$. The elements of $\Lambda(I)$ are written as $\omega=\omega_{1} \ldots \omega_{m} \omega_{m+1} \ldots$ and the elements of $\Lambda_{n}(I)$ are written as words $\omega=\omega_{1} \ldots \omega_{n}$, where $\omega_{i} \in I$. Hence $\Lambda(I)$ is the set of infinite words with letters from the alphabet $I$ and $\Lambda_{n}(I)$ is the set of words of length $n$ with letters from the alphabet $I$. By $\Lambda^{*}(I)$ we denote the set of all finite words, i.e. $\Lambda^{*}(I)=\bigcup_{n \in \mathbb{N}^{*}} \Lambda_{n}(I) \cup\{\lambda\}$, where by $\lambda$ we mean the empty word. If $\omega=\omega_{1} \ldots \omega_{m} \omega_{m+1} \ldots \in \Lambda(I)$ or if $\omega=\omega_{1} \ldots \omega_{n} \in$ $\Lambda_{n}(I)$, where $m, n \in \mathbb{N}^{*}, n \geq m$, then the word $\omega_{1} \ldots \omega_{m}$ is denoted by $[\omega]_{m}$. By $|\omega|$ we mean the length of $\omega$. For two words $\alpha=\alpha_{1} \ldots \alpha_{n} \in \Lambda_{n}(I)$ and $\beta=\beta_{1} \ldots \beta_{m} \in \Lambda_{m}(I)$ or $\beta=\beta_{1} \ldots \beta_{m} \beta_{m+1} \ldots \in \Lambda(I)$, by $\alpha \beta$ we mean the concatenation of the words $\alpha$ and $\beta$, i.e. $\alpha \beta=\alpha_{1} \ldots \alpha_{n} \beta_{1} \ldots \beta_{m}$ and respectively, $\alpha \beta=\alpha_{1} \ldots \alpha_{n} \beta_{1} \ldots \beta_{m} \beta_{m+1} \ldots$ On $\Lambda(I)$, we consider the metric

$$
d_{\Lambda}(\alpha, \beta)=\sum_{k=1}^{\infty} \frac{1-\delta_{\alpha_{k}}^{\beta_{k}}}{3^{k}}, \quad \text { where } \delta_{x}^{y}= \begin{cases}1 & \text { if } x=y, \\ 0 & \text { if } x \neq y,\end{cases}
$$

$\alpha=\alpha_{1} \ldots \alpha_{n+1} \alpha_{n+2} \ldots$ and $\beta=\beta_{1} \ldots \beta_{n+1} \beta_{n+2} \ldots$

Let $(X, d)$ be a metric space and $f_{i}: X \rightarrow X, i \in I$. For $\omega=\omega_{1} \ldots \omega_{m} \in$ $\Lambda_{m}(I)$, we consider $f_{\omega}=f_{\omega_{1}} \circ \ldots \circ f_{\omega_{m}}$ and, for a subset $H$ of $X, H_{\omega}=f_{\omega}(H)$. We also consider $f_{\lambda}=\mathrm{Id}$ and $H_{\lambda}=H$.

For a subset $A$ of a metric space $(X, d)$, we denote by $\operatorname{diam}(A)$ the diameter of $A$ (or, if necessary, $\operatorname{diam}_{d}(A)$ ).

\section{Possibly infinite self-similar systems}

A possibly infinite self-similar system generalizes the concept of the attractor of an infinite iterated function system containing $\varphi$-contractions (see [5] and [15]), as Proposition 3.7 points out. 
Definition 3.1. A possibly infinite self-similar system (PISSS for short) consists of a complete and bounded metric space $(A, d)$ and a family of continuous functions $\left(f_{i}\right)_{i \in I}$, where $f_{i}: A \rightarrow A$, such that:

(a) $A=\overline{\bigcup_{i \in I} A_{i}}$;

(b) $\lim _{n \rightarrow \infty} \sup _{\omega \in \Lambda_{n}(I)} \operatorname{diam}\left(A_{\omega}\right)=0$.

We denote it by $\mathcal{S}=\left((A, d),\left(f_{i}\right)_{i \in I}\right)$. If, in addition, the family of functions $\left(f_{i}\right)_{i \in I}$ is equicontinuous, then $\mathcal{S}$ is called uniformly possibly infinite self-similar system (UPISSS for short).

Definition 3.2. Let $(X, d)$ be a metric space. A family of functions $\left(f_{i}\right)_{i \in I}$, $f_{i}: X \rightarrow X$, is called bounded if the set $\bigcup_{i \in I} f_{i}(A)$ is bounded, for every bounded subset $A$ of $X$.

Definition 3.3. A function $\varphi:[0, \infty) \rightarrow[0, \infty)$ is called a comparison function if it satisfies the following three properties:

(a) $\varphi$ is increasing;

(b) $\varphi(t)<t$ for any $t>0$;

(c) $\varphi$ is right-continuous.

REMARK 3.4. Note that $\varphi(0)=0$ for each comparison function $\varphi$.

REMARK 3.5 (see Remark 1 from [11]). Any function $\varphi:[0, \infty) \rightarrow[0, \infty)$ satisfying (b) and (c) from the above definition has the following property:

$$
\lim _{n \rightarrow \infty} \varphi^{[n]}(t)=0
$$

for any $t>0$, where by $\varphi^{[n]}$ we mean the composition of $\varphi$ by itself $n$ times.

Definition 3.6. Let $(X, d)$ be a metric space and a function $\varphi:[0, \infty) \rightarrow$ $[0, \infty)$. A function $f: X \rightarrow X$ is called $\varphi$-contraction if

$$
d(f(x), f(y)) \leq \varphi(d(x, y)), \quad \text { for every } x, y \in X .
$$

Proposition 3.7. Given a complete metric space $(X, d)$ and a comparison function $\varphi:[0, \infty) \rightarrow[0, \infty)$, if a bounded family of functions $\left(f_{i}\right)_{i \in I}$, where $f_{i}: X \rightarrow X$, is such that each function $f_{i}$ is $\varphi$-contraction, then there exists a unique bounded and closed subset $A$ of $X$ such that $A=\overline{\bigcup_{i \in I} A_{i}}$ and $((A, d)$, $\left.\left(f_{i} \mid A\right)_{i \in I}\right)$ is a UPISSS.

Proof. We have:

(a) For the existence of the set $A$ see Theorem 2.5 from [5].

(b) For each $n \in \mathbb{N}$, we have

$$
\sup _{\omega \in \Lambda_{n}(I)} \operatorname{diam}\left(A_{\omega}\right) \leq \varphi^{[n]}(\operatorname{diam}(A)) .
$$


Consequently, taking into account Remarks 3.4 and 3.5, we get that

$$
\lim _{n \rightarrow \infty} \sup _{\omega \in \Lambda_{n}(I)} \operatorname{diam}\left(A_{\omega}\right)=0 .
$$

(c) Using Remark 3.4, we infer that $d\left(f_{i}(x), f_{i}(y)\right) \leq \varphi(d(x, y)) \leq d(x, y)$, for each $x, y \in A$ and $i \in I$, and we conclude that the family of functions $\left(f_{i}\right)_{i \in I}$ is equicontinuous.

The above proposition provides a large class of UPISSSs. In particular, as the functions $\tau_{i}$ have Lipschitz constant less or equal to $1 / 3,\left(\left(\Lambda(I), d_{\Lambda}\right),\left(\tau_{i}\right)_{i \in I}\right)$ is a UPISSS having the property that $\left(\Lambda(I), d_{\Lambda}\right)$ is not compact, in case that $I$ is infinite.

The next two propositions emphasize a connection between the points of $\Lambda(I)$ and the elements of $A$.

Proposition 3.8. Let $\mathcal{S}=\left((A, d),\left(f_{i}\right)_{i \in I}\right)$ be a PISSS. Then, for each $\omega \in$ $\Lambda(I)$, the set $\bigcap_{n \in \mathbb{N}^{*}} \overline{A_{[\omega]_{n}}}$ has exactly one element.

Proof. Note that $A_{[\omega]_{n+1}} \subseteq A_{[\omega]_{n}}$, so $\overline{A_{[\omega]_{n+1}}} \subseteq \overline{A_{[\omega]_{n}}}$ for each $n \in \mathbb{N}^{*}$ and

$$
\lim _{n \rightarrow \infty} \operatorname{diam}\left(\overline{A_{[\omega]_{n}}}\right)=\lim _{n \rightarrow \infty} \operatorname{diam}\left(A_{[\omega]_{n}}\right)=0 .
$$

Then, since $A$ is a complete metric space, basing on Cantor's intersection theorem, we conclude that $\bigcap_{n \in \mathbb{N}^{*}} \overline{A_{[\omega]_{n}}}$ has one point.

We denote by $a_{\omega}$ the element of $\bigcap_{n \in \mathbb{N}^{*}} \overline{A_{[\omega]_{n}}}$, so $\left\{a_{\omega}\right\}=\bigcap_{n \in \mathbb{N}^{*}} \overline{A_{[\omega]_{n}}}$.

Proposition 3.9. Let $\mathcal{S}=\left((A, d),\left(f_{i}\right)_{i \in I}\right)$ be a PISSS. Then, in the framework of the previous proposition, for each $a \in A$ and each $\omega \in \Lambda$, we have

$$
\lim _{n \rightarrow \infty} f_{[\omega]_{n}}(a)=a_{\omega}
$$

Moreover, the convergence is uniform with respect to a and $\omega$, i.e. for each $\varepsilon>0$ there exists $n_{\varepsilon} \in \mathbb{N}^{*}$ such that the inequality $d\left(f_{[\omega]_{m}}(a), a_{\omega}\right)<\varepsilon$ is valid for each $n \in \mathbb{N}^{*}, n \geq n_{\varepsilon}, a \in A$ and $\omega \in \Lambda(I)$.

Proof. Since, for every $n \in \mathbb{N}^{*}$,

$$
d\left(f_{[\omega]_{n}}(a), a_{\omega}\right) \leq \operatorname{diam}\left(\overline{A_{[\omega]_{n}}}\right)=\operatorname{diam}\left(A_{[\omega]_{n}}\right) \leq \sup _{\omega \in \Lambda_{n}(I)} \operatorname{diam}\left(A_{\omega}\right),
$$

and $\lim _{n \rightarrow \infty} \sup _{\omega \in \Lambda_{n}} \operatorname{diam}\left(A_{\omega}\right)=0$, we infer that for every $a \in A$ and every $\omega \in \Lambda(I)$, we have $\lim _{n \rightarrow \infty} f_{[\omega]_{n}}(a)=a_{\omega}$ uniformly with respect to $a \in A$ and $\omega \in \Lambda(I)$. 


\section{A remetrization that makes contractive all the constitutive functions of a UPISSS}

In this section, given a UPISSS $\left((A, d),\left(f_{i}\right)_{i \in I}\right)$, we construct a metric $\rho$, which is equivalent to $d$, having the property that all the functions $f_{i}:(A, \rho) \rightarrow$ $(A, \rho)$ are contractive.

THEOREM 4.1. Let $\left((A, d),\left(f_{i}\right)_{i \in I}\right)$ be a UPISSS. Then there exists a metric $\rho$ on $A$ having the following three properties:

(a) $\rho\left(f_{i}(x), f_{i}(y)\right) \leq \rho(x, y)$, for each $i \in I$ and each $x, y \in A$; consequently $\rho\left(f_{\omega}(x), f_{\omega}(y)\right) \leq \rho(x, y)$, for each $x, y \in A$ and each $\omega \in \Lambda^{*}(I)$.

(b) $\rho$ is equivalent to $d$.

(c) The metric space $(A, \rho)$ is complete and bounded.

Proof. Define $\rho: A \times A \rightarrow[0, \infty)$ by

$$
\rho(x, y)=\sup _{\omega \in \Lambda^{*}(I)} d\left(f_{\omega}(x), f_{\omega}(y)\right), \quad \text { for every } x, y \in A .
$$

The function $\rho$ attains finite values since $d\left(f_{\omega}(x), f_{\omega}(y)\right) \leq \operatorname{diam}(A)$ for every $\omega \in \Lambda^{*}(I)$ and every $x, y \in A$. Obviously, $\rho$ is a bounded metric in $A$, satisfies (a) and $d \leq \rho$.

To establish (b) we only have to prove that if $\left(a_{n}\right)_{n \in \mathbb{N}}$ is a sequence of elements from $A$ and $l \in A$ is such that $\lim _{n \rightarrow \infty} d\left(a_{n}, l\right)=0$, then $\lim _{n \rightarrow \infty} \rho\left(a_{n}, l\right)=0$.

Indeed, as $\lim _{n \rightarrow \infty} \sup _{\omega \in \Lambda_{n}(I)} \operatorname{diam}\left(A_{\omega}\right)=0$, for every $\varepsilon>0$ there exists $m_{\varepsilon} \in \mathbb{N}$ such that $\sup _{\omega \in \Lambda_{(I)}} \operatorname{diam}\left(A_{\omega}\right)<\varepsilon / 2$ for every $n \in \mathbb{N}, n \geq m_{\varepsilon}$, so

$$
d\left(f_{\omega}\left(a_{n}\right), f_{\omega}(l)\right) \leq \operatorname{diam}\left(A_{\omega}\right) \leq \sup _{\omega^{\prime} \in \Lambda_{|\omega|}(I)} \operatorname{diam}\left(A_{\omega^{\prime}}\right)<\varepsilon / 2
$$

for every $n \in \mathbb{N}$ and every $\omega \in \Lambda^{*}(I)$ with $|\omega| \geq m_{\varepsilon}$. Since the family of functions $\left(f_{i}\right)_{i \in I}$ is equicontinuous, the family $\left(f_{\omega}\right)_{\omega \in \Lambda^{*}(I),|\omega|<m_{\varepsilon}}$ has the same property, so, for every $\varepsilon>0$ there exists $n_{\varepsilon} \in \mathbb{N}$ such that the inequality $d\left(f_{\omega}\left(a_{n}\right), f_{\omega}(l)\right)<$ $\varepsilon / 2$ is valid for every $n \in \mathbb{N}, n \geq n_{\varepsilon}$ and every $\omega \in \Lambda^{*}(I)$ such that $|\omega|<m_{\varepsilon}$. We showed that for every $\varepsilon>0$ there exists $n_{\varepsilon} \in \mathbb{N}$ such that

$$
\rho\left(a_{n}, l\right)=\sup _{\omega \in \Lambda^{*}(I)} d\left(f_{\omega}\left(a_{n}\right), f_{\omega}(l)\right) \leq \varepsilon / 2<\varepsilon
$$

for every $n \in \mathbb{N}, n \geq n_{\varepsilon}$. Hence $\lim _{n \rightarrow \infty} \rho\left(a_{n}, l\right)=0$.

Now we prove (c). The boundedness of $(A, \rho)$ is obvious as $\rho(x, y) \leq \operatorname{diam}(A)$ for every $x, y \in A$. We claim that $(A, \rho)$ is complete.

Indeed, if $\left(a_{n}\right)_{n \in \mathbb{N}}$ is a $\rho$-Cauchy sequence of elements from $A$, then $\left(a_{n}\right)_{n \in \mathbb{N}}$ is also a $d$-Cauchy sequence. As $(A, d)$ is complete, there exists $l \in A$ such that $\lim _{n \rightarrow \infty} d\left(a_{n}, l\right)=0$ and therefore $\lim _{n \rightarrow \infty} \rho\left(a_{n}, l\right)=0$.

Proposition 4.2. In the above framework $\left((A, \rho),\left(f_{i}\right)_{i \in I}\right)$ is a PISSS. 
Proof. According to Theorem $4.1(\mathrm{c}),(A, \rho)$ is complete and bounded.

As the metrics $d$ and $\rho$ are equivalent, the function $f_{i}:(A, \rho) \rightarrow(A, \rho)$ is continuous for each $i \in I$ (since $f_{i}:(A, d) \rightarrow(A, d)$ is continuous) and the equality $A=\overline{\bigcup_{i \in I} A_{i}}$, which is valid for $d$, is also true for $\rho$.

Moreover, for every $x, y \in A, n \in \mathbb{N}$ and $\omega \in \Lambda_{n}(I)$, we have

$$
\begin{array}{r}
\left.\rho\left(f_{\omega}(x), f_{\omega}(y)\right)=\sup _{\theta \in \Lambda^{*}(I)} d\left(f_{\theta}\left(f_{\omega}(x)\right), f_{\theta}\left(f_{\omega}(y)\right)\right)=\sup _{\theta \in \Lambda^{*}(I)} d\left(f_{\theta \omega}(x)\right), f_{\theta \omega}(y)\right) \\
\leq \sup _{\theta \in \Lambda^{*}(I)} \operatorname{diam}_{d}\left(A_{\theta \omega}\right) \leq \sup _{\theta \in \Lambda^{*}(I)} \operatorname{diam}_{d}\left(A_{[\theta \omega]_{n}}\right) \leq \sup _{\omega \in \Lambda_{n}(I)} \operatorname{diam}_{d}\left(A_{\omega}\right),
\end{array}
$$

$\mathrm{SO}$

$$
\sup _{\omega \in \Lambda_{n}(I)} \operatorname{diam}_{\rho}\left(A_{\omega}\right) \leq \sup _{\omega \in \Lambda_{n}(I)} \operatorname{diam}_{d}\left(A_{\omega}\right), \quad \text { for every } n \in \mathbb{N} .
$$

Since $\lim _{n \rightarrow \infty} \sup _{\omega \in \Lambda_{n}(I)} \operatorname{diam}_{d}\left(A_{\omega}\right)=0$, from the previous inequality it follows that $\lim _{n \rightarrow \infty} \sup _{\omega \in \Lambda_{n}(I)} \operatorname{diam}_{\rho}\left(A_{\omega}\right)=0$. We conclude that $\left((A, \rho),\left(f_{i}\right)_{i \in I}\right)$ is a PISSS.

Remark 4.3. According to Propositions 3.9 and 4.2, for each $\varepsilon>0$ there exists $n_{\varepsilon} \in \mathbb{N}^{*}$ such that the inequality $\rho\left(f_{[\omega]_{n}}(a), a_{\omega}\right)<\varepsilon$ is valid for every $n \in \mathbb{N}^{*}, n \geq n_{\varepsilon}, a \in A$ and $\omega \in \Lambda(I)$.

Using the method of mathematical induction, we get a strictly increasing sequence of natural numbers $\left(m_{k}\right)_{k \in \mathbb{N}^{*}}$ such that the inequality

$$
\rho\left(f_{[\omega]_{n}}(a), a_{\omega}\right)<\frac{5^{k-1}}{2^{4 k}}
$$

is valid for every $k \in \mathbb{N}^{*}, n \in \mathbb{N}^{*}, n \geq m_{k}, a \in A$ and $\omega \in \Lambda(I)$.

Note that, using the triangle inequality, we get that

$$
\rho\left(f_{[\omega]_{n}}\left(a_{1}\right), f_{[\omega]_{n}}\left(a_{2}\right)\right)<\frac{5^{k-1}}{2^{4 k-1}}
$$

for each $k \in \mathbb{N}^{*}, n \in \mathbb{N}^{*}, n \geq m_{k}, a_{1}, a_{2} \in A$ and $\omega \in \Lambda(I)$.

\section{A remetrization that makes $\varphi$-contractions all the constitutive functions of a UPISSS}

In this section, given a UPISSS $\left((A, d),\left(f_{i}\right)_{i \in I}\right)$, we construct a comparison function $\varphi$ and a metric $\delta$, which is equivalent to $d$, such that all the functions $f_{i}:(A, \delta) \rightarrow(A, \delta)$ are $\varphi$-contractions.

We mention that in the sequel:

- By $L$ we mean $\lim _{n \rightarrow \infty} L_{n}$, where $L_{n}=\prod_{k=1}^{n+1}\left(1+2^{-k}\right)$ for every $n \in \mathbb{N}$. Note that, since

$$
\ln L_{n}=\ln \prod_{k=1}^{n+1}\left(1+2^{-k}\right)=\sum_{k=1}^{n+1} \ln \left(1+2^{-k}\right) \leq \sum_{k=1}^{n+1} 2^{-k}<1
$$


for every $n \in \mathbb{N}$, the sequence $\left(L_{n}\right)_{n \in \mathbb{N}}$ is bounded. As it is clear that it is also increasing, we infer that it is convergent.

- $x_{k}=5^{k-1} / 2^{4 k-1}$ for every $k \in \mathbb{N}^{*}$.

- $\left(m_{k}\right)_{k \in \mathbb{N}^{*}}$ is the sequence from Remark 4.3 and $y_{k}=L_{m_{k}} / L_{m_{k}}+1=$ $2^{m_{k}+2} / 2^{m_{k}+2}+1$ for every $k \in \mathbb{N}^{*}$.

Given a UPISSS $\left((A, d),\left(f_{i}\right)_{i \in I}\right)$, we consider the function $\delta: A \times A \rightarrow[0, \infty]$ given by

$$
\delta(x, y)=\sup _{\omega \in \Lambda^{*}(I)} L_{|\omega|} \rho\left(f_{\omega}(x), f_{\omega}(y)\right),
$$

for every $x, y \in A$, where $\rho$ is the metric introduced in Theorem 4.1.

Proposition 5.1. In the above framework, the inequality

$$
\frac{3}{2} \rho(x, y) \leq \delta(x, y) \leq L \rho(x, y),
$$

is valid for every $x, y \in A$.

Proof. On the one hand, for every $x, y \in A$, we have

$$
\frac{3}{2} \rho(x, y)=L_{|\lambda|} \rho\left(f_{\lambda}(x), f_{\lambda}(y)\right) \leq \delta(x, y) .
$$

On the other hand, since by Theorem 4.1 (a) the inequality

$$
L_{|\omega|} \rho\left(f_{\omega}(x), f_{\omega}(y)\right) \leq L_{|\omega|} \rho(x, y) \leq L \rho(x, y)
$$

is valid for every $\omega \in \Lambda^{*}(I), x, y \in A$, we get that

$$
\delta(x, y)=\sup _{\omega \in \Lambda^{*}(I)} L_{|\omega|} \rho\left(f_{\omega}(x), f_{\omega}(y)\right) \leq L \rho(x, y),
$$

for every $x, y \in A$.

Hence $\delta: A \times A \rightarrow[0, \infty)$ and it is a metric which is equivalent to $\rho$, so to $d$, as the reader can routinely verify.

Proposition 5.2. In the above framework, the inequality

$$
\delta\left(f_{i}(x), f_{i}(y)\right) \leq \delta(x, y),
$$

is valid for every $x, y \in A$ and every $i \in I$.

Proof. We have

$$
\begin{aligned}
& L_{|\omega|} \rho\left(f_{\omega}\left(f_{i}(x)\right), f_{\omega}\left(f_{i}(y)\right)\right)=L_{|\omega|} \rho\left(f_{\omega i}(x), f_{\omega i}(y)\right) \\
& \quad \leq L_{|\omega i|} \rho\left(f_{\omega i}(x), f_{\omega i}(y)\right) \leq \sup _{\omega \in \Lambda^{*}(I)} L_{|\omega|} \rho\left(f_{\omega}(x), f_{\omega}(y)\right)=\delta(x, y)
\end{aligned}
$$

for every $x, y \in A, i \in I$ and $\omega \in \Lambda^{*}(I)$, so

$$
\delta\left(f_{i}(x), f_{i}(y)\right)=\sup _{\omega \in \Lambda^{*}(I)} L_{|\omega|} \rho\left(f_{\omega}\left(f_{i}(x)\right), f_{\omega}\left(f_{i}(y)\right)\right) \leq \delta(x, y),
$$

for every $x, y \in A$ and every $i \in I$. 
Proposition 5.3. In the above framework, the inequality

$$
\delta\left(f_{i}(x), f_{i}(y)\right) \leq \max \left\{\sup _{\omega \in \Lambda^{*}(I),|\omega|<m_{k}} L_{|\omega|} \rho\left(f_{\omega i}(x), f_{\omega i}(y)\right), L x_{k}\right\}
$$

is valid for every $x, y \in A, i \in I$ and $k \in \mathbb{N}^{*}$.

Proof. We have

$$
\begin{aligned}
& \delta\left(f_{i}(x), f_{i}(y)\right)=\sup _{\omega \in \Lambda^{*}(I)} L_{|\omega|} \rho\left(f_{\omega}\left(f_{i}(x)\right), f_{\omega}\left(f_{i}(y)\right)\right) \\
= & \max \left\{\sup _{\omega \in \Lambda^{*}(I),|\omega|<m_{k}} L_{|\omega|} \rho\left(f_{\omega i}(x), f_{\omega i}(y)\right), \sup _{\omega \in \Lambda^{*}(I),|\omega| \geq m_{k}} L_{|\omega|} \rho\left(f_{\omega i}(x), f_{\omega i}(y)\right)\right\} \\
& \operatorname{Remark}^{\operatorname{Rem} 4.3} \max \left\{\sup _{\omega \in \Lambda^{*}(I),|\omega|<m_{k}} L_{|\omega|} \rho\left(f_{\omega i}(x), f_{\omega i}(y)\right), L x_{k}\right\},
\end{aligned}
$$

for every $x, y \in A, i \in I$ and $k \in \mathbb{N}^{*}$.

Proposition 5.4. In the above framework, for every $k \in \mathbb{N}, x, y \in A$ and $i \in I$, we have $\delta\left(f_{i}(x), f_{i}(y)\right) \leq y_{k} \delta(x, y)$, provided that $L x_{k}<\delta\left(f_{i}(x), f_{i}(y)\right)$.

Proof. Let us consider $k \in \mathbb{N}, x, y \in A$ and $i \in I$ such that $L x_{k}<$ $\delta\left(f_{i}(x), f_{i}(y)\right)$. Then, taking into account Proposition 5.3, we have

$$
L x_{k}<\delta\left(f_{i}(x), f_{i}(y)\right) \leq \max \left\{\sup _{\omega \in \Lambda^{*}(I),|\omega|<m_{k}} L_{|\omega|} \rho\left(f_{\omega i}(x), f_{\omega i}(y)\right), L x_{k}\right\},
$$

so,

$$
\delta\left(f_{i}(x), f_{i}(y)\right) \leq \sup _{\omega \in \Lambda^{*}(I),|\omega|<m_{k}} L_{|\omega|} \rho\left(f_{\omega i}(x), f_{\omega i}(y)\right) .
$$

Then, for every $\varepsilon>0$ there exists $\omega_{\varepsilon} \in \Lambda^{*}(I),\left|\omega_{\varepsilon}\right|<m_{k}$ such that

$$
\delta\left(f_{i}(x), f_{i}(y)\right)-\varepsilon<L_{\left|\omega_{\varepsilon}\right|} \rho\left(f_{\omega_{\varepsilon} i}(x), f_{\omega_{\varepsilon} i}(y)\right)
$$

and consequently, as the sequence $\left(L_{n} / L_{n+1}\right)_{n \in \mathbb{N}^{*}}$ is increasing, we get

$$
\delta\left(f_{i}(x), f_{i}(y)\right)-\varepsilon<L_{\left|\omega_{\varepsilon} i\right|} \rho\left(f_{\omega_{\varepsilon} i}(x), f_{\omega_{\varepsilon} i}(y)\right) \frac{L_{\left|\omega_{\varepsilon}\right|}}{L_{\left|\omega_{\varepsilon} i\right|}} \leq \frac{L_{\left|\omega_{\varepsilon}\right|}}{L_{\left|\omega_{\varepsilon} i\right|}} \delta(x, y) \leq y_{k} \delta(x, y),
$$

for every $\varepsilon>0$. Therefore $\delta\left(f_{i}(x), f_{i}(y)\right) \leq y_{k} \delta(x, y)$.

THEOREM 5.5. Let $\left((A, d),\left(f_{i}\right)_{i \in I}\right)$ be a UPISSS. Then there exist a comparison function $\varphi$ and a metric $\delta$, which is equivalent to $d$, such that

$$
\delta\left(f_{i}(x), f_{i}(y)\right) \leq \varphi(\delta(x, y)),
$$

for every $x, y \in A$ and $i \in I$, i.e. the function $f_{i}:(A, \delta) \rightarrow(A, \delta)$ is $\varphi$-contraction for every $i \in I$. 
Proof. Note that, in the above framework, the strictly decreasing sequence $\left(x_{k}\right)_{k \in \mathbb{N}^{*}}$ of positive reals converges to 0 and the strictly increasing sequence $\left(y_{k}\right)_{k \in \mathbb{N}^{*}}$ of reals greater or equal to $1 / 2$ converges to 1 .

With the notation $z_{k}=2 L x_{k}$, one can easily check that, for every $k \in \mathbb{N}^{*}$, $z_{k} \leq z_{k-1} / 2$ and $z_{k} y_{k+1} \leq z_{k-1} y_{k}$. Moreover, we have

$(*) \forall i \in I \quad \forall k \in \mathbb{N}^{*} \quad \forall x, y \in A \quad \delta(x, y)>z_{k} \Rightarrow \delta\left(f_{i}(x), f_{i}(y)\right) \leq y_{k} \delta(x, y)$.

Indeed, if $\delta\left(f_{i}(x), f_{i}(y)\right) \leq z_{k} / 2$, then we have $\delta\left(f_{i}(x), f_{i}(y)\right) \leq z_{k} / 2<\delta(x, y) / 2$ $\leq y_{k} \delta(x, y)$ and if $\delta\left(f_{i}(x), f_{i}(y)\right)>z_{k} / 2$, we just use Proposition 5.4.

Now we define the function $\varphi:[0, \infty) \rightarrow[0, \infty)$ in the following way:

$$
\varphi(0)=0, \quad \varphi(t)=t-z_{1}\left(1-y_{2}\right)
$$

for $t \in\left(z_{1}, \infty\right)$ and

$$
\varphi(t)=\left(\frac{t-z_{k}}{z_{k-1}-z_{k}}\right) z_{k-1} y_{k}+\left(\frac{z_{k-1}-t}{z_{k-1}-z_{k}}\right) z_{k} y_{k+1},
$$

for every $k \in \mathbb{N}, k \geq 2$ and every $t \in\left(z_{k}, z_{k-1}\right]$. It is clear that $\varphi$ is a comparison function.

Now we prove that $\delta\left(f_{i}(x), f_{i}(y)\right) \leq \varphi(\delta(x, y))$, for every $x, y \in A$ and $i \in I$. Since the above inequality is obvious if $\delta(x, y)=0$, we shall treat the following two cases:

(i) $\delta(x, y) \in\left(z_{1}, \infty\right)$;

(ii) $\delta(x, y) \in\left(z_{k}, z_{k-1}\right]$ for some $k \in \mathbb{N}, k \geq 2$.

In the first case, from $(*)$, we infer that $\delta\left(f_{i}(x), f_{i}(y)\right) \leq y_{1} \delta(x, y)$ for every $i \in I$. As $z_{1}<\delta(x, y)$ and $y_{1} \leq y_{2}$, we obtain

$$
y_{1} \delta(x, y) \leq \delta(x, y)-z_{1}\left(1-y_{2}\right)=\varphi(\delta(x, y))
$$

and we conclude that $\delta\left(f_{i}(x), f_{i}(y)\right) \leq \varphi(\delta(x, y))$ for every $i \in I$.

In the second case, using again $(*)$, we get $\delta\left(f_{i}(x), f_{i}(y)\right) \leq y_{k} \delta(x, y)$ for every $i \in I$. As $z_{k}<\delta(x, y) \leq z_{k-1}$, we obtain

$$
y_{k} \delta(x, y) \leq\left(\frac{\delta(x, y)-z_{k}}{z_{k-1}-z_{k}}\right) z_{k-1} y_{k}+\left(\frac{z_{k-1}-\delta(x, y)}{z_{k-1}-z_{k}}\right) z_{k} y_{k+1}=\varphi(\delta(x, y)),
$$

and we conclude that $\delta\left(f_{i}(x), f_{i}(y)\right) \leq \varphi(\delta(x, y))$ for every $i \in I$.

Definition 5.6. Given a metric space $(X, d)$, a possibly infinite iterated function system is a pair $\mathcal{S}=\left((X, d),\left(f_{i}\right)_{i \in I}\right)$, where $f_{i}: X \rightarrow X$ is continuous for every $i \in I$.

Definition 5.7. Given a comparison function $\varphi:[0, \infty) \rightarrow[0, \infty)$, a possibly infinite iterated function system $\mathcal{S}=\left((X, d),\left(f_{i}\right)_{i \in I}\right)$ is called $\varphi$-hyperbolic if there exists a metric $\delta$ on $X$, equivalent to $d$, such that the function $f_{i}:(X, \delta) \rightarrow$ $(X, \delta)$ is $\varphi$-contraction for every $i \in I$. 
Now, Theorem 5.5 could be restated in the following way:

THEOREM 5.8. Let $\left((A, d),\left(f_{i}\right)_{i \in I}\right)$ be a UPISSS. Then there exists a comparison function $\varphi$ such that the possibly infinite iterated function system $\mathcal{S}=$ $\left((A, d),\left(f_{i}\right)_{i \in I}\right)$ is $\varphi$-hyperbolic.

REMARK 5.9. Taking into account Proposition 3.7, which states that each possibly infinite iterated function system whose constitutive functions form a bounded family of $\varphi$-contractions generates a uniformly possibly infinite selfsimilar system, and Theorem 5.8, that says that for each uniformly possibly infinite self-similar system there exists a comparison function $\varphi$ such that it becomes a $\varphi$-hyperbolic possibly infinite iterated function system, we conclude that there exists a strong connection between uniformly possibly infinite selfsimilar systems and $\varphi$-hyperbolic possibly infinite iterated function systems.

\section{Kameyama's topological self-similar systems are particular cases of possibly infinite self-similar systems}

Proposition 6.1. In the framework of Definition 1.1, we have

$$
K=\overline{\bigcup_{i=1}^{N} K_{i}}
$$

Proof. Indeed, for each $x \in K=\pi(\Lambda)$ there exists $\omega=\omega_{1} \ldots \omega_{m} \omega_{m+1} \ldots$ in $\Lambda$ such that $x=\pi(\omega)=\pi\left(\omega_{1} \omega^{\prime}\right)=f_{\omega_{1}}\left(\pi\left(\omega^{\prime}\right)\right) \in K_{\omega_{1}}$, where $\omega^{\prime}=\omega_{2} \ldots \omega_{m+1} \ldots$, so $x \in \bigcup_{i=1}^{N} K_{i}$. Thus $K \subseteq \bigcup_{i=1}^{N} K_{i} \subseteq K$, so $K=\bigcup_{i=1}^{N} K_{i}$. As $K$ is compact, we infer that $K=\overline{\bigcup_{i=1}^{N} K_{i}}$.

TheOREm 6.2 (see [10, Theorem 5.1]). A topological self-similar set is metrizable.

Proposition 6.3 (see [10, Lemma 1.6]). Let $\left(K,\left\{f_{i}\right\}_{i \in\{1, \ldots, N\}}\right)$ be a topological self-similar system and $d$ any metric on $K$ which is compatible with the original topology. Then

$$
\lim _{n \rightarrow \infty}\left(\max _{\omega \in \Lambda_{n}(\{1, \ldots, N\})} \operatorname{diam}\left(K_{\omega}\right)\right)=0 .
$$

REMARK 6.4. From Proposition 6.1 and Proposition 6.3, we infer that if $\left(K,\left\{f_{i}\right\}_{i \in\{1, \ldots, N\}}\right)$ is a topological self-similar system and $d$ any metric on $K$ which is compatible with the original topology, then $\mathcal{S}=\left((K, d),\left(f_{i}\right)_{i \in I}\right)$, where $I=\{1, \ldots, N\}$, is a PISSS. In addition, since the functions $f_{i}$ are continuous and the set $I$ is finite, $\mathcal{S}$ is a UPISSS. Consequently Kameyama's topological self-similar systems are particular cases of possibly infinite self-similar systems 
and Theorem 5.5 is a generalization of our result from [13] stating that given a topological self-similar system $\left(K,\left(f_{i}\right)_{i \in\{1, \ldots, N\}}\right)$ there exist a metric $\delta$ on $K$ which is compatible with the original topology and a comparison function $\varphi$ such that $f_{i}:(K, \delta) \rightarrow(K, \delta)$ is $\varphi$-contraction for every $i \in\{1, \ldots, N\}$.

Now let us consider a PISSS $\mathcal{S}=\left((A, d),\left(f_{i}\right)_{i \in I}\right)$ for which the set $I$ is finite.

Proposition 6.5. In the above framework, $(A, d)$ is a compact Hausdorff topological space.

Proof. From the definition of a PISSS we have $A=\overline{\bigcup_{i \in I} A_{i}}$, so

$$
A_{j}=f_{j}\left(\bigcup_{i \in I} \overline{A_{i}}\right)=\bigcup_{i \in I} f_{j}\left(\overline{A_{i}}\right) \stackrel{f_{j}}{\subseteq} \stackrel{\text { continuous }}{\subseteq} \overline{f_{j}\left(A_{i}\right)}=\bigcup_{i, j \in I} \overline{A_{j i}}
$$

for every $j \in I$. Hence $A=\overline{\bigcup_{j \in I} A_{j}} \subseteq \bigcup_{i, j \in I} \overline{A_{j i}} \subseteq A$, so $A=\bigcup_{\omega \in \Lambda_{2}(I)} \overline{A_{\omega}}$. In a similar way we can prove that

$$
A=\bigcup_{\omega \in \Lambda_{n}(I)} \overline{A_{\omega}} \text { for every } n \in \mathbb{N} .
$$

As $\lim _{n \rightarrow \infty} \sup _{\omega \in \Lambda_{n}(I)} \operatorname{diam}\left(\overline{A_{\omega}}\right)=\lim _{n \rightarrow \infty} \sup _{\omega \in \Lambda_{n}(I)} \operatorname{diam}\left(A_{\omega}\right)=0$ and $\Lambda_{n}(I)$ is finite, from $(*)$ we infer that $A$ is totally bounded. Since it is also complete, we conclude that it is compact.

Theorem 5.5 assures us that there exist a comparison function $\varphi$ and a metric $\delta$, which is equivalent to $d$, such that all the functions $f_{i}:(A, \delta) \rightarrow(A, \delta)$ are $\varphi$-contractions. Since $A=\bigcup_{i \in I} f_{i}(A)$, we come to the conclusion that the attractor of the iterated function system $\left((A, \delta),\left(f_{i}\right)_{i \in I}\right)$ is $A$. Note that, taking into account Proposition 3.8, we can consider the function $\pi: \Lambda(I) \rightarrow A$ given by

$$
\pi(\omega)=a_{\omega} \quad \text { for every } \omega \in \Lambda(I) .
$$

Then, from the standard properties of such iterated function systems (see, for example, [6], where the more general case of iterated function systems consisting of Meir-Keeler functions is treated) we obtain the following result:

Proposition 6.6. In the above framework, the function $\pi$ has the following properties:

(a) it is onto;

(b) $\pi \circ \tau_{i}=f_{i} \circ \pi$ for every $i \in I$;

(c) it is continuous.

Remark 6.7. From Propositions 6.5 and 6.6, we conclude that a PISSS $\mathcal{S}=\left((A, d),\left(f_{i}\right)_{i \in I}\right)$ for which the set $I$ is finite is a topological self-similar system, hence the concepts of PISSS and topological self-similar system coincide for finite sets $I$. 
Acknowledgements. We want to thank the referees whose generous and valuable remarks and comments brought improvements to the paper and enhanced clarity.

\section{REFERENCES}

[1] R. Atrins, M. Barnsley, A. Vince And D. Wilson, A characterization of hyperbolic affine iterated function systems, Topology Proc. 36 (2010), 189-211.

[2] T. Banakh, W. Kubiś, N. Novosad, M. Nowak and F. Strobin, Contractive function systems, their attractors and metrization, Topol. Methods Nonlinear Anal. 46 (2015), 1029-1066 .

[3] M. Barnsley and K. IGudesman, Topological contractive systems, Lobackevskii J. Math. 32 (2011), 220-223.

[4] M. F. Barnsley And A. Vince, Real projective iterated function systems, J. Geom. Anal. 22 (2012), 1137-1172.

[5] D. Dumitru, Attractors of infinite iterated function systems containing contraction type functions, An. Ştiinţ. Univ. Al. I. Cuza Iaşi, Mat. N.S. 59 (2013), 281-298.

[6] D. Dumitru And A. Minail, The shift space of an iterated function system containing Meir-Keeler functions, An. Univ. Bucureşti Mat. 57 (2008), 75-88.

[7] K. Hveberg, Injective mapping systems and self-homeomorphic fractals, Ph.D. Thesis, University of Oslo, 2005.

[8] L. Janoš, A converse of the Banach's contraction theorem, Proc. Amer. Math. Soc. 18 (1967), 287-289.

[9] L. Janoš, H.-M. Ko And K.-K. TAN, Edelstein's contractivity and attractors, Proc. Amer. Math. Soc. 76 (1979), 339-344.

[10] A. Kameyama, Distances on topological self-similar sets and the kneading determinants, J. Math. Kyoto Univ. 40 (2000), 603-674.

[11] J. Matkowski, Fixed point theorems for mappings with a contractive iterate at a point, Proc. Amer. Math. Soc. 62 (1977), 344-348.

[12] R. Miculescu and A. Minail, Alternative characterization of hyperbolic affine infinite iterated function systems, J. Math. Anal. Appl. 407 (2013), 56-68.

[13] , On a question of A. Kameyama concerning self-similar metrics, J. Math. Anal. Appl. 422 (2015), 265-271.

[14] A. Vince, Möbius iterated function systems, Trans. Amer. Math. Soc. 365 (2013), 491509.

[15] K. Wicks, Fractals and hyperspaces, Lecture Notes in Math. 1492, Springer-Verlag, 1991.

Manuscript received September 7, 2014

accepted March 24, 2015

Radu Miculescu and Alexandru Mihail

Faculty of Mathematics and Computer Science

University of Bucharest

Str. Academiei 14

010014 Bucharest, ROMANIA

E-mail address: miculesc@yahoo.com,mihail_alex@yahoo.com

TMNA : Volume $47-2016-\mathrm{N}^{\mathrm{O}} 1$ 